\title{
Reaction waves in solid fuels for adiabatic competitive exothermic reactions
}

\author{
Z. Huang ${ }^{1} \quad$ H. S. Sidhu ${ }^{2} \quad$ I. N. Towers ${ }^{3}$ \\ Z. Jovanoski ${ }^{4}$ V. V. Gubernov ${ }^{5}$
}

(Received 26 February 2015; revised 10 November 2015)

\begin{abstract}
We investigate travelling premixed reaction waves in a diffusionalthermal model with a two-step competitive reaction mechanism where both reactions are exothermic. Travelling waves are assumed to propagate at constant speed. An approximation of the Arrhenius reaction rate is adopted to simplify the combustion model. Based on this assumption, an asymptotic theory is presented for solid fuels under adiabatic conditions. This approach provides a convenient way to analyse the system in the phase plane. The asymptotic speeds for the flame fronts are compared with numerical solutions by solving the governing partial differential equations. In addition, piecewise approximate solutions for the temperature and fuel mass fraction profiles are presented and
\end{abstract}

http://journal.austms.org.au/ojs/index.php/ANZIAMJ/article/view/9343 gives this article, (C) Austral. Mathematical Soc. 2015. Published December 15, 2015, as part of the Proceedings of the 17th Biennial Computational Techniques and Applications Conference. ISSN 1446-8735. (Print two pages per sheet of paper.) Copies of this article must not be made otherwise available on the internet; instead link directly to this URL for this article. 
compared with those obtained numerically. Our results can be applied to combustion synthesis in the production of advanced materials.

\section{Contents}

1 Introduction

C149

2 Governing equations

C150

3 Asymptotic analysis

C153

4 Piecewise approximation

C156

5 Conclusion

C158

References

C160

\section{Introduction}

This work is concerned with the existence and propagation of reaction fronts through reactive media in the presence of diffusive processes with two-step reaction kinetics. We assume that both reactions are exothermic. Such reactions have direct relevance to the combustion of $\mathrm{MeCH}_{2}$, where Me is either Ti or Zr [1, 2]. Sidhu et al. [3] and Towers et al. [4] investigated flame propagation in a model with two-stage competitive exothermic reactions (exoexo reactions) and demonstrated the existence of regions of bi-stability - where stable solutions corresponding to slow and fast branches co-exist. Regions of pulsating combustion waves were also reported by these authors.

Besides the two-step competitive exothermic reactions mentioned above, a number of authors investigated reaction schemes where a main exothermic reaction is accompanied by an endothermic reaction [5, 6, 7]. Hmaidi et al. [5] 
investigated the existence and stability of travelling one-dimension reaction fronts propagating through a solid reactive slab (infinite Lewis number), extending the work of Matkowshy and Sivashinsky [6] by including the effects of heat loss through a competitive endothermic reaction. Forbes [7] proposed an asymptotic approximation to the nonlinear temperature-dependent reaction rates. A phase plane analysis was presented for this reaction scheme under both adiabatic and non-adiabatic conditions. The results for the approximate and numerical wave speeds show very close correspondence.

Here we present an analysis of a two-step competitive exo-exo reaction scheme, using a similar approach to Forbes [7]. The approximation to the exponential term of the Arrhenius function has two advantages: it avoids the "cold boundary' difficulty - the reactions occur as long as the temperature is greater than absolute zero, which is not true in reality; and it simplifies the model without increasing the number of dimensionless parameters. A phase plane analysis is given for the evolutionary system for solid fuels under adiabatic conditions. Numerical solutions, obtained by solving the full governing partial differential equations using two independent approaches, are employed to validate the asymptotic result. Additionally, piecewise approximate solutions for the fuel and temperature profiles are presented. We also compare these approximate solutions with those obtained via a standard shooting method and with the solutions of the governing partial differential equations (PDEs).

\section{Governing equations}

Consider a model with a two-step competitive reaction mechanism arranged in a one-dimensional configuration, where both reactions are thermally and chemically coupled. A general schematic for this process is

$$
A \stackrel{k_{1}(T)}{\longrightarrow} B+Q_{1}, \quad A \stackrel{k_{2}(T)}{\longrightarrow} C+Q_{2},
$$

where $A$ represents the reactant; $B$ and $C$ are chemically inert products under different conditions which do not change their physical properties, 
such as density, heat capacity or the diffusivity of the system; $\mathrm{Q}_{1}$ describes the heat released by the first reaction and $\mathrm{Q}_{2}$ is the heat released by the second reaction. Here we assume neither $\mathrm{Q}_{1}$ nor $\mathrm{Q}_{2}$ is less than zero since only the two-stage competitive exo-exo reaction scheme is of interest in this investigation. The two reaction rates $k_{1}(T)$ and $k_{2}(T)$, assumed to be dependent on the reaction temperature $T$, obey Arrhenius kinetics and have the form $k_{i}(T)=Z_{i} e^{-E_{i} /(R T)}$, where $Z_{i}$ and $E_{i}$ are the first-order rates and the activation energies, respectively, for the respective reaction $i=1,2$. The universal gas constant is $R$.

Following Towers et al. [4], the dimensionless governing equations derived from the conservation of energy and fuel mass are

$$
\begin{aligned}
& \frac{\partial u}{\partial \tau}=\frac{\partial^{2} u}{\partial \xi^{2}}+v k_{1}(u)+q r v k_{2}(u) \\
& \frac{\partial v}{\partial \tau}=\frac{1}{\operatorname{Le}} \frac{\partial^{2} v}{\partial \xi^{2}}-\beta v k_{1}(u)-r \beta v k_{2}(u) .
\end{aligned}
$$

In these equations, the dimensionless temperature and mass fraction are $u$ and $\nu$, respectively; and $\xi$ and $\tau$ represent non-dimensional space and time coordinates, respectively. Also, $q=Q_{1} / Q_{2}$ and $r=Z_{1} / Z_{2}$. The exothermicity parameter $\beta$ is the ratio of activation energy to heat released by the first reaction. The Lewis number Le is the ratio of thermal conductivity to mass diffusivity, varying from around unity for gaseous fuels to infinity for solid fuels [8].

Using the Arrhenius law to describe reaction rates has come under criticism by several researchers [7, e.g.]. The main issue is that this approach implies reactions occur at any temperature above absolute zero - the "cold boundary 
difficulty" [6, 8]. Adopting the approach of Forbes [7], the reaction rates are

$$
\begin{aligned}
& k_{1}(u)= \begin{cases}0, & u<\theta, \\
1-\exp [f(\theta-u)], & u>\theta,\end{cases} \\
& k_{2}(u)= \begin{cases}0, & u<\theta, \\
1-\exp (\theta-u), & u>\theta,\end{cases}
\end{aligned}
$$

where $f=E_{1} / E_{2}$ and $\theta$ is the ignition temperature. The form of the reaction rates (3) is more realistic than the Arrhenius form used elsewhere [3, 4, 5, 6] as no reaction should occur when the fuel temperature is below the ignition temperature. Considering the leading term only, the Taylor series of the two reactions in equation (3) with center $\mathfrak{u}_{0}=\theta$ are

$$
k_{1}(u) \approx\left\{\begin{array} { l l } 
{ 0 , } & { u < \theta , } \\
{ f ( u - \theta ) , } & { u > \theta , }
\end{array} \quad k _ { 2 } ( u ) \approx \left\{\begin{array}{ll}
0, & u<\theta \\
u-\theta, & u>\theta
\end{array}\right.\right.
$$

A new moving coordinate frame $\eta=\xi-c \tau$ is introduced where the constant $c$ is the speed of travelling waves. Consequently, the governing PDEs are transformed into a system of ordinary differential equations (ODEs). Then we simplify the governing equations by using (4) without increasing the number of dimensionless parameters by setting $u \triangleq u-\theta$. Thus, the governing PDEs (2) are transformed to two second-order ODEs,

$$
\begin{aligned}
& -\mathrm{cu}^{\prime}=\mathrm{u}^{\prime \prime}+(1+\mathrm{qrf}) v \mathrm{u}, \\
& -\mathrm{c} v^{\prime}=\frac{1}{\mathrm{Le}} v^{\prime \prime}-(1+\mathrm{rf}) \beta v \mathrm{u},
\end{aligned}
$$

with the dash denoting ordinary differentiation with respect to $\eta$. The evolutionary system (5) obeys the boundary conditions

$$
\begin{array}{lll}
u=\theta_{a}, & v=v_{0}, & \eta \rightarrow \infty, \\
u_{\eta}=0, & v_{\eta}=0, & \eta \rightarrow-\infty .
\end{array}
$$


The right boundary condition $(\eta \rightarrow \infty)$ is a 'cold' and 'unburnt' state (the dimensionless ambient temperature is $\theta_{a}$ and the consumption of the fuel is negligible). For definiteness and without loss of generality, the initial mass fraction $v_{0}$ is chosen to be one. However, on the left boundary $(\eta \rightarrow-\infty)$, neither the temperature nor the mass fraction can be specified. No reaction is supposed to occur at this steady state. Therefore, the derivatives of $u$ and $v$ are set to zero for $\eta \rightarrow-\infty$.

\section{$3 \quad$ Asymptotic analysis}

In this section, travelling combustion wave solutions are sought to equation (5) for solid fuels (the material diffusion is negligible and thus the Lewis number is treated as infinite). The relevant second-order governing ODEs for solid fuels are

$$
\begin{aligned}
& -c u^{\prime}=u^{\prime \prime}+(1+q r f) v u, \\
& -c v^{\prime}=-(1+r f) \beta v u .
\end{aligned}
$$

Substituting (8) into (7) leads to

$$
-c u^{\prime}=u^{\prime \prime}+(1+q r f) c v^{\prime} /(1+r f) \beta .
$$

Integrating (9) once and utilizing the 'cold' and 'unreacted' upstream boundary condition $(u=0, v=1$ as $\eta \rightarrow \infty)$ yields

$$
v=1-\left(u^{\prime}+c u\right) / c \lambda,
$$

where $\lambda=(1+$ qrf $) /(1+r f) \beta$. Substituting (10) back into (7) to eliminate $v$ leads to

$$
u^{\prime \prime}+\left(c-\frac{(1+q r f) u}{c \lambda}\right) u^{\prime}+(1+q r f) u\left(1-\frac{u}{\lambda}\right)=0
$$


Equation (11) has two uniform stationary solutions: $\mathfrak{u}=0$ and $\mathfrak{u}=\lambda$. Thus, it is reasonable to assume the solutions to equation (11) satisfy the boundary conditions

$$
\begin{aligned}
& u \rightarrow \lambda, \quad v \rightarrow 0, \quad \eta \rightarrow-\infty \\
& u \rightarrow 0, \quad v \rightarrow 1, \quad \eta \rightarrow+\infty
\end{aligned}
$$

The right boundary condition $(\eta \rightarrow+\infty)$ is a 'cold' and 'unburnt' state, whereas the left $(\eta \rightarrow-\infty)$ corresponds to the 'hot' and 'burnt' state. The temperature at the left boundary $\lambda$ must be greater than zero for practical interest. The ignition temperature $\theta$ can be any value not less than zero. Hence, for definiteness and without loss of generality, the value is set to zero.

Equation (11) is amenable to analysis using the phase plane approach. We rewrite (11) as a system of two first-order ordinary differential equations:

$$
\frac{\mathrm{du}}{\mathrm{d} \eta}=\mathrm{F}, \quad \frac{\mathrm{dF}}{\mathrm{d \eta}}=-\left[\mathrm{c}-\frac{(1+\mathrm{qrf}) \mathrm{u}}{\mathrm{c} \lambda}\right] \mathrm{F}-(1+\mathrm{qrf}) \mathrm{u}\left(1-\frac{\mathrm{u}}{\lambda}\right) .
$$

To investigate the properties of the travelling waves, we linearize the system about the two uniform steady states. For the stationary state $(u, F)=(0,0)$, ahead of the flame front, the linearization of (13) yields the eigenvalues

$$
\mu_{1,2}^{+}=\frac{-c \pm \sqrt{c^{2}-4(1+q r f)}}{2}
$$

with the corresponding eigenvectors $k_{1,2}^{+}=\left(1, \mu_{1,2}^{+}\right)^{\top}$. For real eigenvalues we require $c \geqslant 2 \sqrt{1+\mathrm{qrf}}$. The system (11) is of the Fisher-type, therefore the physically realizable solution is the one with minimal speed (Fisher [7] and Tikhomirov [8] provide details),

$$
c=2 \sqrt{1+q r f} .
$$

The stationary point $u=0$ is a degenerate stable node where the wave speed is given by (15). Similarly, we can determine the eigenvalues and the corresponding eigenvectors at $u=\lambda$. 
Figure 1: Temperature and mass fraction profiles for a travelling wave with parameter values $q=1, r=1 / 2, f=3 / 2, \beta=1, v_{0}=1$ and $\theta_{0}=0$. The red solid line is the temperature profile and the blue dash line is the profile of the fuel mass fraction.

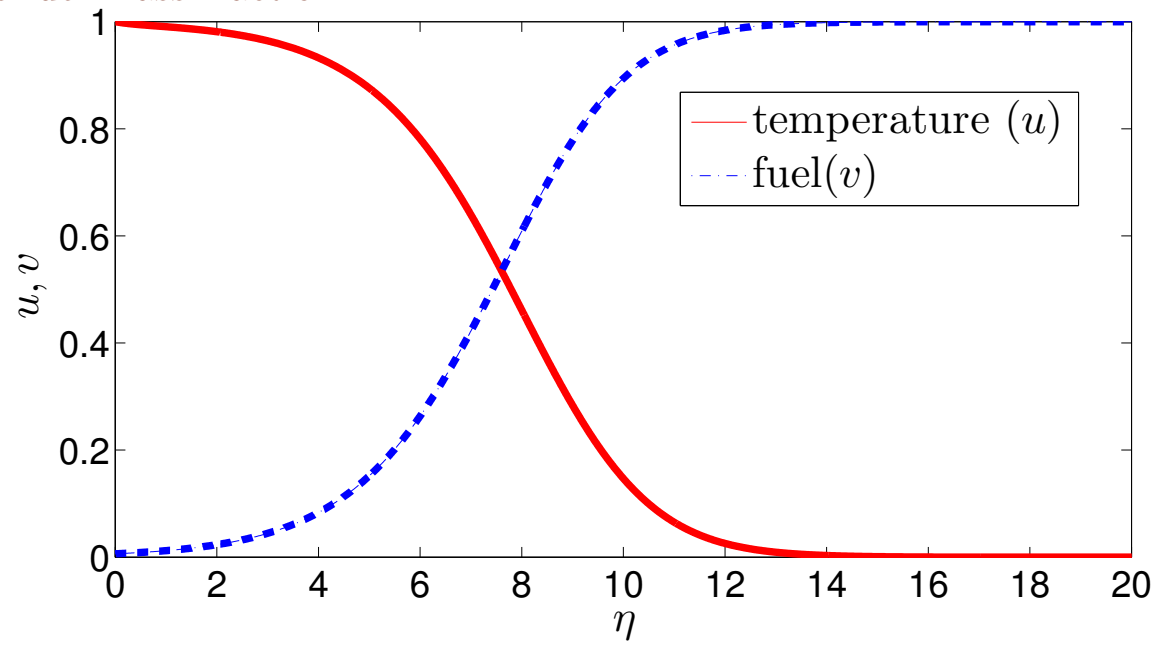

For illustrative purposes, we provide some sample results with parameter values $q=1, r=1 / 2, f=3 / 2, \beta=1$, and the mass fraction is initially $v_{0}=1$, while the initial conditions for temperature are $\theta_{0}=\theta=0$. The temperature profile is obtained using a standard shooting method to solve (13). Once the temperature profile is determined, the mass fraction profile is obtained via relationship (10). The profiles of mass fraction and temperature are shown in Figure 1. The temperature profile decreases monotonically from the maximum value $u=1$ (behind the front) to the ambient temperature (ahead of the front). The fuel mass fraction profile increases monotonically from the burnt value zero (behind the front) to the 'unburnt' value $v_{0}=1$ (ahead of the front).

To validate the asymptotic results presented above we utilize two independent methods to solve the governing PDE system (2) with the rate functions (3): the method of lines (MOL) [10] and a commercial finite element package 
FlexPDE $^{\mathrm{TM}}$ [4]. The initial mass fraction was set to the maximum value $v(x, 0)=1$, whereas the temperature was initially taken to be a simple Gaussian profile, $\mathbf{u}=A \exp \left(-\chi^{2}\right)$ where $A$ is a constant. Varying $A$ has no impact on the numerical results presented here other than the length of computational time that is required to obtain a steady propagating combustion wave. When using the MOL, a uniform grid of 1501 mesh nodes was placed along the $x$-axis over the interval $0<x<300$, and the time span was $t \in[0,100]$. The average speed obtained was $c \approx 2.642$. Increasing the spatial and time intervals to $0<x<4000$ and $t \in[0,1000]$ with 40001 nodes resulted in the average speed of the wave $c \approx 2.638$. When using the commercial finite-element package FlexPDE ${ }^{\mathrm{TM}}$, the average speeds for the wave were found to be $\mathrm{c} \approx 2.656$ and $\mathrm{c} \approx 2.639$, where the error tolerances were set to $10^{-6}$ and $10^{-7}$, respectively. The asymptotic speed calculated from (15) is $\mathbf{c}=2.6458$. Therefore, the results from the two independent numerical approaches provide a validation for the asymptotic results of the adiabatic exo-exo scheme for solid fuels.

\section{Piecewise approximation}

In this section we obtain piecewise approximate solutions for the temperature and fuel mass fraction profiles by replacing the non-linear terms in the governing differential equation by a set of linear segments tangent to the original curve [9]. Here we focus on the special case $c=2 \sqrt{1+\mathrm{qrf}}$. We consider the simplest case of two segments. From our earlier analysis we know that the critical point with $u=0$ is a degenerate node and has eigenvalues $\mu_{ \pm}=-c / 2$. Furthermore, we also know that the stationary point with $u=\lambda$ has eigenvalues $r_{+}=c / 4$ and $r_{-}=-c$. Following Jovanoski and Robinson [9] we write the two-segment approximate solution to (11) as

$$
u(\eta)= \begin{cases}\lambda+C e^{c \eta / 4}+D e^{-c \eta}, & \eta \leqslant \eta_{0}, \\ A e^{-c \eta / 2}+B \eta e^{-c \eta / 2}, & \eta \geqslant \eta_{0} .\end{cases}
$$


Since the solution is supposed to satisfy the asymptotic behaviour $u \rightarrow \lambda$ when $\eta \rightarrow-\infty$, we require $D=0$. Due to translational invariance, we specify $\eta_{0}=0$ and $\mathfrak{u}(0)=\lambda / 2$. Using the continuity of $\mathfrak{u}(\eta)$ and of the derivative $u^{\prime}(\eta)$ at $\eta_{0}$ yields $A=c / 2, B=c \lambda / 8$ and $C=-\lambda / 2$. Thus, the complete solution for (16) is

$$
u(\eta)= \begin{cases}\lambda-\frac{1}{2} \lambda e^{c \eta / 4}, & \eta \leqslant 0, \\ \frac{1}{2} \lambda e^{-c \eta / 2}+\frac{1}{8} c \lambda \eta e^{-c \eta / 2}, & \eta \geqslant 0 .\end{cases}
$$

Once $u(\eta)$ is obtained, the approximate solution for mass fraction $y(\eta)$ is determined using the relationship (10). Hence we obtain

$$
y(\eta)= \begin{cases}\frac{5}{8} \lambda e^{c \eta / 4}, & \eta \leqslant 0 \\ 1-\frac{3}{8} e^{-c \eta / 2}-\frac{1}{16} c \eta e^{-c \eta / 2}, & \eta \geqslant 0\end{cases}
$$

We extend the basic two-segment method outlined above to determine a piecewise function with three segments to approximate the solution of system (11). To do this we divide the domain into three subintervals $\left(-\infty, \eta_{1}\right]$, $\left[\eta_{1}, \eta_{2}\right]$ and $\left[\eta_{2}, \infty\right)$. In particular, we specify $\eta_{0}=0, u(0)=\lambda / 2$ and $\eta_{0} \in\left[\eta_{1}, \eta_{2}\right]$. Then, the approximate solution for the temperature is

$$
u(\eta)= \begin{cases}\lambda+C e^{c \eta / 4}+D e^{-c \eta}, & \eta \leqslant \eta_{1}, \\ \lambda / 2+u_{3}(\eta), & \eta_{1} \leqslant \eta \leqslant \eta_{2}, \\ A e^{-c \eta / 2}+B \eta e^{-c \eta / 2}, & \eta>\eta_{2}\end{cases}
$$

Substituting $\boldsymbol{u}$ into (11) over the domain $\left[\eta_{1}, \eta_{2}\right]$, the ODE with respect to $\boldsymbol{u}_{3}$ is

$$
u_{3}^{\prime \prime}+\left(c-\frac{1+k}{2 c}\right) u_{3}^{\prime}+\frac{(1+k) \lambda}{4}=0
$$

where $k=1+q r f$. The ODE above has the analytical solution

$$
u_{3}(\eta)=E-\frac{(1+k) \lambda \eta}{4[c-(1+k) / 2 c]}+F e^{-\eta[c-(1+k) / 2 c]}
$$


where $E$ and $F$ are constants. To determine the approximate solution using three segments, the quantities $A, B, C, D, E, F, \eta_{1}$ and $\eta_{2}$, must be determined. Requiring $u \rightarrow 0$ as $\eta \rightarrow-\infty$ implies $D=0$. We also assume that the solution is twice continuously differentiable over the entire domain. The continuity of $u, u^{\prime}$ and $u^{\prime \prime}$ at the matched points $\eta=\eta_{1}, \eta=\eta_{2}$ yields six equations. Due to translational invariance, we specify $u(0)=\lambda / 2$. Thus, we establish seven equations to solve seven unknown parameters. The system of seven equations has the unique solution

$$
\begin{aligned}
& A=0.3187 \lambda, \quad B=0.8500 \lambda, \quad C=-0.6250 \lambda, \quad E=-F, \\
& F=-0.0005 \lambda, \quad \eta_{1}=-1.5633, \quad \eta_{2}=1.1364 .
\end{aligned}
$$

Having determined $\mathfrak{u}(\eta)$, the fuel mass fraction profile $\boldsymbol{y}(\eta)$ is obtained via (10). The profiles for the temperature and mass fraction are shown in Figures 2 and 3, respectively. As well as the approximate solutions, the numerical solutions obtained by the standard shooting method solving (11), and the MOL solutions from the governing PDEs (2) with the reaction rates (4) are also shown. Both of these numerical approaches result in solutions that correspond well with each other (maximum difference being around $10^{-3}$ ). The two-segment and three-segment approximate solutions agree with asymptotic behaviours as $\eta \rightarrow \pm \infty$. However, both Figures 2 and 3 clearly show that although the two-segment approach provides a reasonable solution, the threesegment approximation is much better over the entire domain. Compared with the numerical solutions, the maximum error of the two-segment approximation is around $12 \%$, whereas the maximum error falls sharply to around $3 \%$ when using the three-segment approach.

\section{Conclusion}

We utilize the asymptotic approximate method of Forbes [7] to study combustion waves for a two-stage competitive exo-exo reaction scheme for solid fuels 
Figure 2: The temperature profile for a travelling wave with the same parameter values as in Figure 1. Results are obtained using the shooting method (red solid line), the MOL on PDEs (2) with (4) (green circles), and the two-segment (blue dashed line) and three-segment (black dot line) approximate solutions.

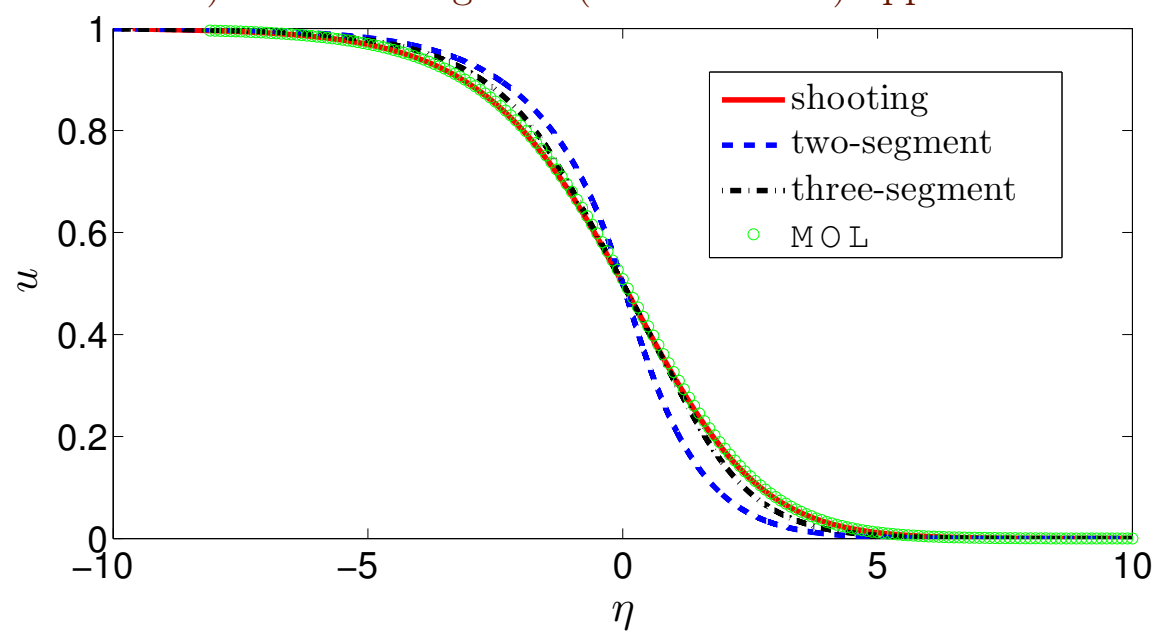

Figure 3: The fuel mass fraction profile. The notation, symbols and parameter values are the same as in Figure 2.

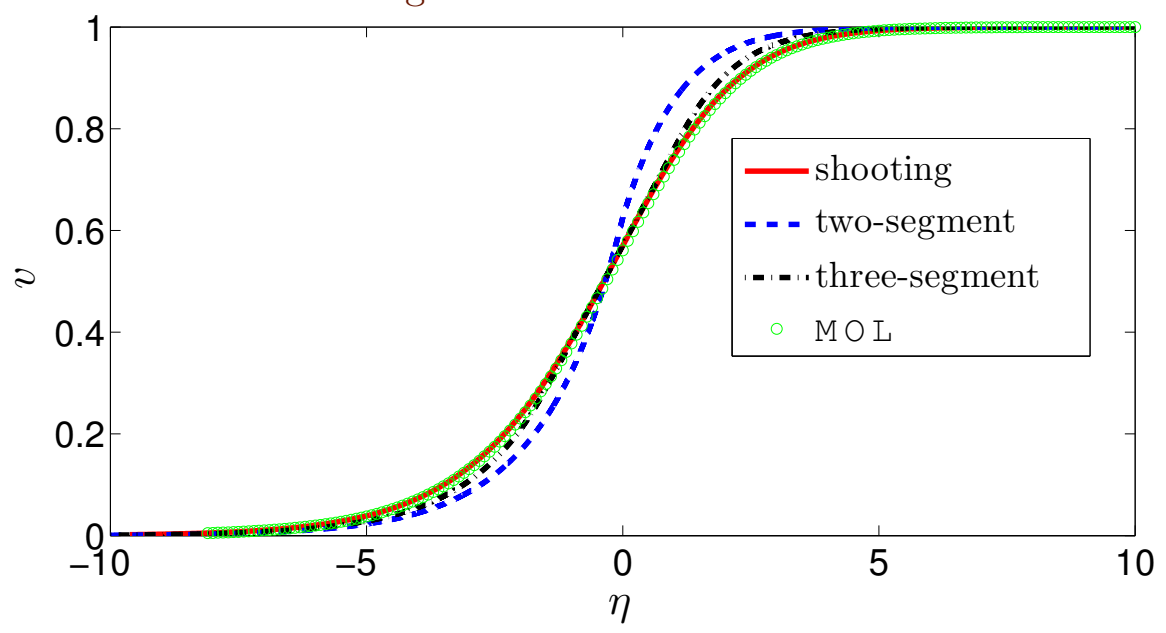


under adiabatic conditions. By approximating the Arrhenius-type reaction rates with the leading term, the system reduces to a simple second-order ODE which is easily analysed in the phase-plane. By undertaking such an analysis we obtain an explicit form for the speed of the reaction wave. Despite the 'crude' approximation used, the asymptotic results obtained for the speeds of the reaction waves show remarkable agreement with numerical solutions obtained by solving the governing PDEs (2) with rate functions (3) using two independent approaches. Similar agreement for the wave speeds was observed by Forbes [7] for the competitive endothermic-exothermic reaction scheme.

In this study we also used the piecewise linear approximation to obtain the temperature and fuel mass fraction profiles. Once again the results show considerable accuracy when compared to the numerical solutions obtained using two different approaches. Future work will focus on using these approximate methods for the non-adiabatic case and for fuels other than solid fuels.

\section{References}

[1] Martirosyan, N. A., Dolukhanyan, S. K., and Merzhanov, A. G., Experimental observation of the nonuniqueness of stationary combustion in systems with parallel reactions. Combust. Explos. Shock Waves 19(6):711-712, 1983. doi:10.1007/BF00750777 C149

[2] Martirosyan, N. A., Dolukhanyan, S. K., and Merzhanov, A. G., Nonuniqueness of stationary states in combustion of mixtures of zirconium and soot powders in hydrogen. Combust. Explos. Shock Waves 19(5):569-571, 1983. doi:10.1007/BF00750423 C149

[3] Sidhu, H. S., Towers, I. N., Gubernov, V. V., Kolobov, A. V., and Polezhaev, A. A., Investigation of flame propagation in a model with competing exothermic reactions. Chemeca 2013, Brisbane, Australia, 29 September-2 October, 2013. 
http: //www . conference. net . au/chemeca2013/papers/29350.pdf C149, C152

[4] Towers, I. N., Gubernov, V. V., Kolobov, A. V., Polezhaev, A. A., and Sidhu, H. S., Bistability of flame propagation in a model with competing exothermic reactions. P. R. Soc. Lond. A Mat. 469:2158, 2013. doi:10.1098/rspa.2013.0315 C149, C151, C152, C156

[5] Hmaidi, A., McIntosh, A. C., and Brindley, J., A mathematical model of hotspot condensed phase ignition in the presence of a competitive endothermic reaction. Combust. Theory Model. 14(6):893-920, 2010. doi:10.1080/13647830.2010.519050 C149, C152

[6] Matkowsky, B. J., and Sivashinsky, G. I., Propagation of a pulsating reaction front in solid fuel combustion. SIAM J. Appl. Math. 35(3):465-478, 1978. doi:10.1137/0135038 C149, C150, C152

[7] Forbes, L. K., A note on travelling waves in competitive reaction systems. ANZIAM J. 55(1):1-13, 2013. doi:10.1017/S1446181113000333 C149, C150, C151, C152, C154, C158, C160

[8] Barenblatt, G., The Mathematical theory of combustion and explosions. Springer, 1985. http://www.springer.com/us/book/9781461294399 C151, C152, C154

[9] Jovanoski, Z., and Robinson, G., Piecewise linear approximation to Fisher's equation. ANZIAM J. 53:C465-C477, 2011. http://journal. austms.org.au/ojs/index.php/ANZIAMJ/article/view/5129 C156

[10] Schiesser, W. E., The numerical method of lines: integration of partial differential equations. Academic Press, 1991. C155 


\section{Author addresses}

1. Z. Huang, Applied and Industrial Mathematics Research Group, School of Physical, Environmental and Mathematical Sciences, University of New South Wales at the Australian Defence Force Academy, Canberra, Australia.

mailto: zhe .huang@student. adfa.edu.au

2. H. S. Sidhu, Applied and Industrial Mathematics Research Group, School of Physical, Environmental and Mathematical Sciences, University of New South Wales at the Australian Defence Force Academy, Canberra, Australia.

3. I. N. Towers, Applied and Industrial Mathematics Research Group, School of Physical, Environmental and Mathematical Sciences, University of New South Wales at the Australian Defence Force Academy, Canberra, Australia.

4. Z. Jovanoski, Applied and Industrial Mathematics Research Group, School of Physical, Environmental and Mathematical Sciences, University of New South Wales at the Australian Defence Force Academy, Canberra, Australia.

5. V. V. Gubernov, I.E. Tamm Theory Department, P.N. Lebedev Physical Institute, 53 Leninsky Prospect, 119991 Moscow, Russia. 